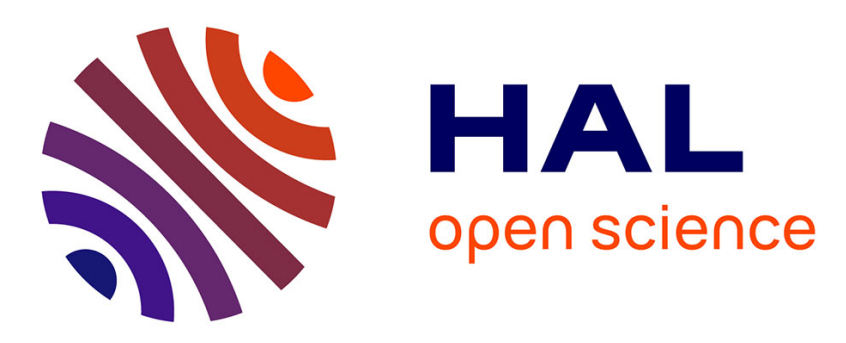

\title{
Phase-space jets drive transport and anomalous resistivity
}

Maxime Lesur, P.H. Diamond, Y. Kosuga

\section{To cite this version:}

Maxime Lesur, P.H. Diamond, Y. Kosuga. Phase-space jets drive transport and anomalous resistivity. Physics of Plasmas, 2014, 21, pp.112307. 10.1063/1.4902525 . hal-01969769

\section{HAL Id: hal-01969769 \\ https://hal.science/hal-01969769}

Submitted on 14 Jan 2019

HAL is a multi-disciplinary open access archive for the deposit and dissemination of scientific research documents, whether they are published or not. The documents may come from teaching and research institutions in France or abroad, or from public or private research centers.
L'archive ouverte pluridisciplinaire HAL, est destinée au dépôt et à la diffusion de documents scientifiques de niveau recherche, publiés ou non, émanant des établissements d'enseignement et de recherche français ou étrangers, des laboratoires publics ou privés. 


\section{AIP $\mid \begin{aligned} & \text { Physiss of } \\ & \text { Plasmas }\end{aligned}$}

\section{Phase-space jets drive transport and anomalous resistivity}

M. Lesur, P. H. Diamond, and Y. Kosuga

Citation: Physics of Plasmas (1994-present) 21, 112307 (2014); doi: 10.1063/1.4902525

View online: http://dx.doi.org/10.1063/1.4902525

View Table of Contents: http://scitation.aip.org/content/aip/journal/pop/21/11?ver=pdfcov

Published by the AIP Publishing

\section{Articles you may be interested in}

Phase space coherent structure of charged particles system

Phys. Plasmas 18, 062107 (2011); 10.1063/1.3590870

The Vlasov-Poisson model and the validity of a numerical approach

Phys. Plasmas 13, 082102 (2006); 10.1063/1.2215596

Anomalous resistivity of current-driven isothermal plasmas due to phase space structuring

Phys. Plasmas 13, 082304 (2006); 10.1063/1.2209611

Nonlinear finite-Larmor-radius drift-kinetic equation

Phys. Plasmas 12, 112305 (2005); 10.1063/1.2116867

Theory of ion holes associated with electron-acoustic vortices

Phys. Plasmas 7, 3247 (2000); 10.1063/1.874190

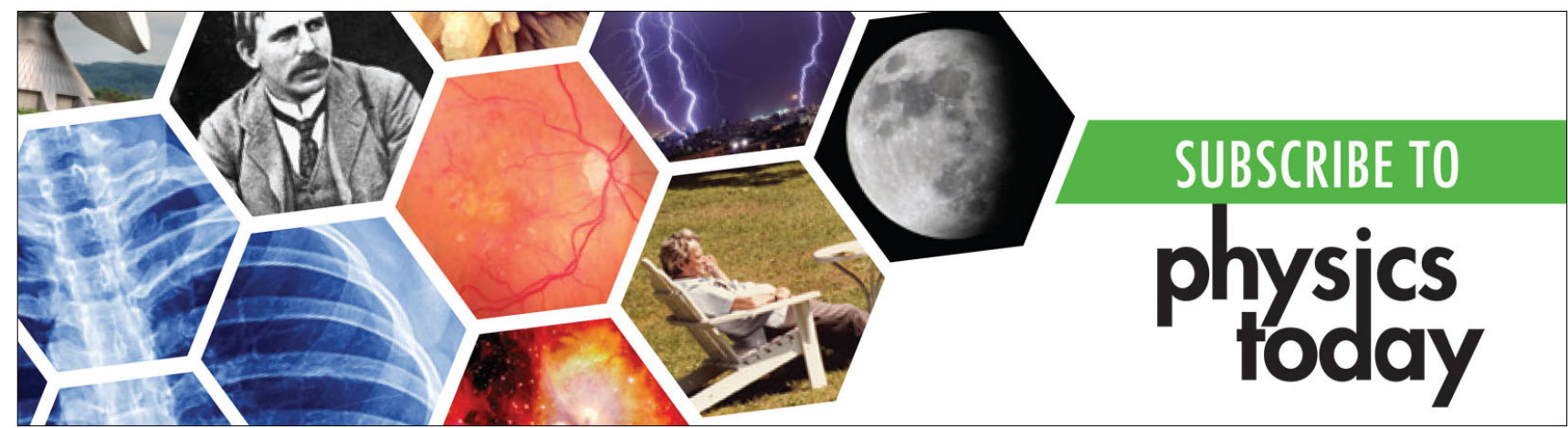




\title{
Phase-space jets drive transport and anomalous resistivity
}

\author{
M. Lesur, ${ }^{1,2, a)}$ P. H. Diamond, ${ }^{3,4}$ and Y. Kosuga ${ }^{1,5}$ \\ ${ }^{1}$ Research Institute for Applied Mechanics, Kyushu University, 6-1 Kasuga Koen, Kasuga 816-8580, Japan \\ ${ }^{2}$ Research Center for Plasma Turbulence, Kyushu University, 6-1 Kasuga Koen, Kasuga 816-8580, Japan \\ ${ }^{3}$ WCI Center for Fusion Theory, NFRI, Gwahangno 113, Daejeon 305-333, Korea \\ ${ }^{4}$ CMTFO and CASS, UCSD, La Jolla, California 92093, USA \\ ${ }^{5}$ Institute for Advanced Study and Research Institute for Applied Mechanics, Kyushu University, \\ Fukuoka 816-8580, Japan
}

(Received 30 April 2014; accepted 12 November 2014; published online 24 November 2014)

In the presence of wave dissipation, phase-space structures spontaneously emerge in nonlinear Vlasov dynamics. These structures include not only well-known self-trapped vortices (holes) but also elongated filaments, resembling jets, as reported in this work. These jets are formed by straining due to interacting holes. Jets are highly anisotropic, and connect low and high velocity regions over a range larger than the electron thermal velocity. Jets survive long enough for particles to scatter between low and high phase-space density regions. Jets are found to contribute significantly to electron redistribution, velocity-space transport, and anomalous resistivity. (C) 2014 AIP Publishing LLC. [http://dx.doi.org/10.1063/1.4902525]

\section{INTRODUCTION}

In this paper, we report on the characteristics of a new type of structure in the now classic collisionless VlasovPoisson turbulence system, and its impacts on relaxation and anomalous resistivity.

The nonlinear evolution of collisionless or weakly collisional plasmas is often accompanied by the formation and ballistic propagation of self-trapped vortices in phase-space. ${ }^{1}$ These coherent structures are spontaneously formed in phasespace by resonant nonlinear wave-particle interactions, which trap particles in a trough. These trapped particles in turn generate a self-potential, leading to a self-sustained structure, which can break ties from resonance. Self-trapped structures resemble vortices in 2D ideal fluid turbulence, ${ }^{2}$ and so reflect similarities between 2D fluids governed by Euler's equation and 1D plasmas governed by Vlasov's equation. Both these systems conserve phase-space density. The evolution of each system is constrained by two invariants: energy and enstrophy in the fluid case, and energy and total phasestrophy $\int f^{2} \mathrm{~d} x \mathrm{~d} v$ in the Vlasov case. ${ }^{3}$ Phase-space vortices, called as electron and ion holes, ${ }^{4}$ open a new channel for tapping free energy in the system. They are key nonlinear agents of instability, ${ }^{5}$ anomalous transport, ${ }^{6}$ and anomalous resistivity, ${ }^{7}$ in the context of energetic particle-driven activities in space and magnetic fusion plasmas, ${ }^{8}$ collisionless magnetic reconnection, ${ }^{9}$ collisionless shock waves, ${ }^{10}$ and alpha-channeling. ${ }^{11}$ Much progress in the still-evolving topic of kinetic nonlinearities is based on the paradigm of phase-space turbulence, ${ }^{12-15}$ where the system is treated as an ensemble of structures in phasespace, rather than an ensemble of waves, as in quasi-linear theory. We can contrast phase-space turbulence with conventional approaches in terms of the Kubo number $K=\omega_{b} \tau_{c}$, which measures the coherence of turbulence. Here, $\omega_{b}$ is the bounce frequency of trapped particles, and $\tau_{c}$ is the correlation

\footnotetext{
a)maxime.lesur@polytechnique.org
}

time of a structure. Conventional theories that rely on linear waves and their nonlinear extensions (mode coupling, weak and strong turbulence theories) require $K<1$ for their validity. This condition is easily violated when wave-particle interactions are strong. Phase-space turbulence theory concerns the ubiquitous $K \gtrsim 1$ regime

Existing phase-space turbulence literature focuses on holes ${ }^{5,16-24}$ and granulations. ${ }^{25,26}$ In this paper, we report on the discovery and effects of a new kind of phase-space structure, called as jet. Jets are highly anisotropic structures, with an extent in velocity of the order of the electron thermal velocity. They are formed by straining due to interacting holes. Therefore, jets are not independent objects. They are tied to the formation and dynamics of holes. This process is similar to the formation of a bridge of material between two colliding galaxies. Indeed, there is a duality between gravitating matter and negative electron phase-space perturbations. ${ }^{27}$ Though less coherent than holes, jets survive long enough for particles to scatter between low and high phase-space density regions. In other words, they efficiently connect low and high velocity regions of phase-space, and so drive significant transport and anomalous resistivity.

Time-scales of holes and jets are ordered as follows: jet lifetime $\sim$ particle travel time on a jet $\sim$ hole trapping time $\ll$ hole lifetime. The lengthscale of jets is of the order of 10 Debye length. The velocity extents are ordered as follows: jet-driven convective acceleration $\lesssim$ hole size $\lesssim$ particle mean-free-path on a jet $\sim$ jet size $\sim$ electron thermal velocity. Jets and holes are associated with fundamentally different transport processes. Hole-driven transport is essentially convective, since trapped particles accelerate along with the hole. Jet-driven transport is essentially stochastic, since particles may accelerate or decelerate along the jet. In terms of magnitude, holes and jets each account for roughly half of the total particle flux.

We develop the example of current-driven ion-acoustic turbulence in a two-species, one-dimensional (1D), 
collisionless electron-ion plasma. In addition to academic interest, a 1D model is relevant for plasma immersed in a strong, relatively homogeneous magnetic field. ${ }^{28}$ In this context, Boutros-Ghali and Dupree derived the nonlinear growth rate of holes ${ }^{26}$ based on earlier and more general work by Dupree. ${ }^{5}$ Then Berman conducted numerical experiments, ${ }^{19,29}$ which validated the theory. Later, Berk et $a l .{ }^{21}$ developed a theory to predict the dynamics of holes and the saturation amplitude in the steady-state, albeit not for current-driven systems. We emphasize that the present work does not focus on holes, but on a new kind of structure, which emerges from hole-plasma or hole-hole interactions.

\section{MODEL}

We apply a standard kinetic model for ion-acoustic turbulence in a two-species, one-dimensional (1D), collisionless electron-ion plasma. The evolution of each particle distribution, $f_{s}(x, v, t)$, where $s=i, e$, is given by the Vlasov equation

$$
\frac{\partial f_{s}}{\partial t}+v \frac{\partial f_{s}}{\partial x}+\frac{q_{s} E}{m_{s}} \frac{\partial f_{s}}{\partial v}=0
$$

where $q_{s}$ and $m_{s}$ are the particle charge and mass, respectively. The electric field $E=-\partial \varphi / \partial x$ satisfies Poisson equation

$$
\frac{\partial E}{\partial x}=\sum_{s} \frac{m_{s} \omega_{p_{s}}^{2}}{n_{0} q_{s}} \int f_{s}(x, v, t) \mathrm{d} v,
$$

where $\omega_{p, s}$ is the plasma frequency and $n_{0}$ is the spatiallyaveraged plasma density.

The initial velocity distribution for each species is a Gaussian, $f_{0, s}(v)=n_{0} /\left[(2 \pi)^{1 / 2} v_{T, s}\right] \exp \left(-\left(\left(v-v_{0, s}\right) / v_{T, s}\right)^{2} / 2\right)$, with $v_{0, i}=0$, and $v_{0, e}=v_{d}$ is the initial drift velocity. The ion and electron temperatures are equal.

We solve this initial-value problem with the semiLagrangian kinetic code COBBLES. ${ }^{30}$ Boundary conditions are periodic in real space, and zero-particle flux at the velocity cutoffs $v_{\text {cut }, s}=v_{0, s} \pm 10 v_{T, s}$. In a one-dimensional periodic system, a spatially uniform current drives a uniform electric field, which oscillates at a frequency $\omega_{u}=\omega_{p, e}\left(1+m_{e} / m_{i}\right)^{1 / 2}$. This rapid oscillation of both the uniform electric field and the uniform current is of little interest here. ${ }^{31}$ Numerically, the average part of $E$ is set to zero, following common practice. . $^{19,32,33}$

We adopt a standard definition of anomalous resistivity, $\eta=E / J$, where $J=e\left(u_{i}-u_{e}\right)$ is the current, and $u_{s}$ is the fluid velocity of species $s$. Combining the electron and ion equations of motion in the collisionless limit yields $\mathrm{d}\left(u_{i}-u_{e}\right)$ / $\mathrm{d} t=\left(m_{i}^{-1}+m_{e}^{-1}\right) e E$. Accordingly, in our code, we estimate anomalous resistivity as $\eta=-m_{i} m_{e} /\left(m_{i}+m_{e}\right) \operatorname{d} \log \left(u_{i}-u_{e}\right) / \mathrm{d} t$.

\section{SMALL MASS RATIO}

In this section, we analyze a numerical experiment with a mass ratio of $m_{i} / m_{e}=4$. The main reason is that a small mass ratio improves numerical tractability and the readability of phase-space contour plots. In addition, it can be argued that low mass ratios are relevant to some laboratory and space plasmas, such as pair plasmas ${ }^{34}$ or electron-positron

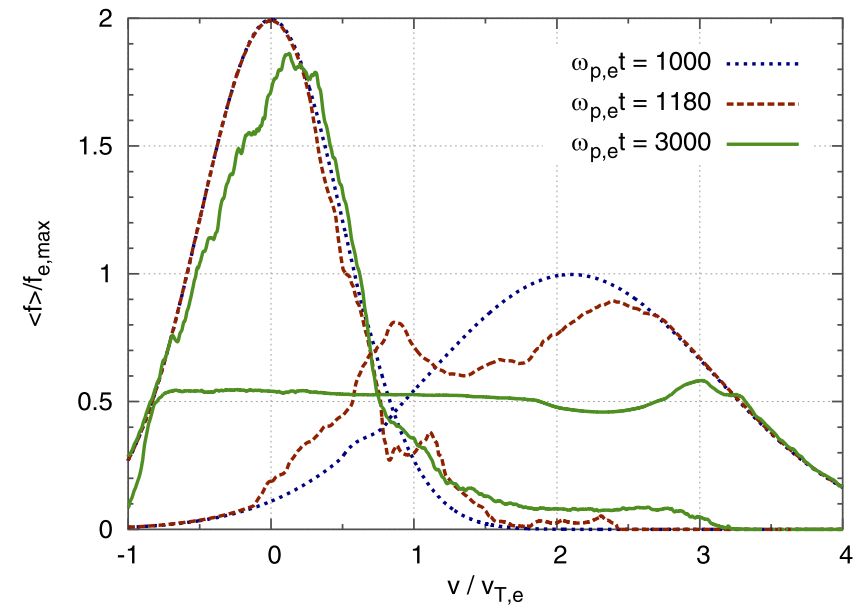

FIG. 1. Snapshots of the ion (left) and electron (right) velocity distributions (mass ratio $m_{i} / m_{e}=4$. System size $L=10 \pi \lambda_{D}$ ).

plasmas. ${ }^{35}$ The analysis is repeated in Sec. IV for a mass ratio of $m_{i} / m_{e}=1836$. Most findings are valid for both mass ratios.

In this section, the system size is $L=2 \pi / k_{1}$, where $k_{1}=0.2 \lambda_{D}^{-1}$. The initial drift is $v_{d}=2.1 v_{T, e}$. The simulation is performed with $N_{x}=1024$ and $N_{v}=2048$ grid points in configuration-space and velocity-space, respectively, and with a time-step width at most $\Delta t=0.016 \omega_{p, e}^{-1}$.

Fig. 1 shows snapshots of the velocity distribution before, during, and after particle redistribution. Electrons loose momentum, which reduces the driving current. Ion redistribution is much weaker. Note that an electron hole at $v / v_{T, e} \approx 2.2$ survives for a long time. It is unfaltering even at the end of the simulation $\left(\omega_{p, e} t=10000\right)$. This is reminiscent of the weakly dissipative coherent trapped-particle state. ${ }^{36}$ Here, dissipation is due to numerical discretization. Fig. 2 shows the evolution, after the linear growth phase, of the mean square potential, $\phi=\left\langle\varphi^{2}\right\rangle^{1 / 2}$, and anomalous resistivity $\eta$. Anomalous resistivity, or equivalently, redistribution of electrons and velocity-space particle transport, is strongest around $t \approx 1150-1400$ (in units of $\omega_{p, e}$ ), which is when coherent structures dominate the phase-space evolution.

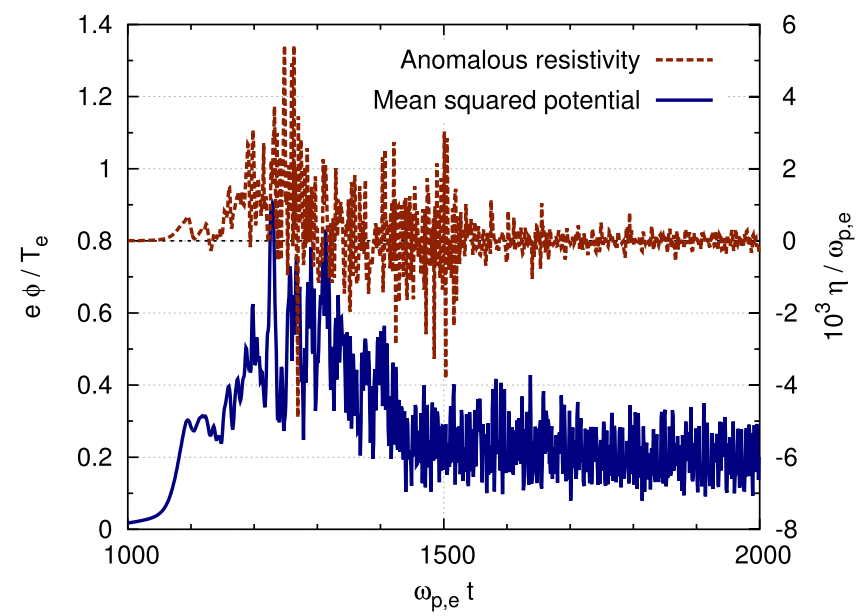

FIG. 2. Time-evolution of the mean square potential (left axis) and anomalous resistivity (right axis). The mass ratio is $m_{i} / m_{e}=4$. The system size is $10 \pi \lambda_{D}$ 
Fig. 3(a) is a snapshot at $t=1180$ of the perturbed electron distribution, $\tilde{f}_{e} \equiv f_{e}-\left\langle f_{e}\right\rangle$, where angled brackets denote an average over real space. Consistent with theory ${ }^{37}$ and earlier simulations, ${ }^{19}$ we observe in the nonlinear phase that phasespace holes spontaneously form in the region of strong overlap between ion and electron distributions. The holes accelerate and grow by climbing the velocity gradient in the electron distribution. Particles that are trapped inside a hole are convected along with it, leading to velocity-space transport. All of the above physics was already documented in the literature.

However, holes are not the only entities which populate the phase-space of particle distributions. We also observe elongated structures, such as the one highlighted in Fig. 3(a) by a curve of constant phase-space density. Since $f_{s}$ is constant along particle orbits, this curve represents an instantaneous electron trajectory. We refer to these structures as jets when they satisfy the three following characteristic properties:

P1. their anisotropy is much higher than that of holes,

P2. they connect low and high velocity regions, separated by $\Delta v_{\text {jet }} \sim v_{T, e}$,

P3. their lifetime $\tau_{\text {jet }}$ is of the same order or higher than the average time $\tau_{\text {travel }}$ it takes a particle to change its velocity by $v_{T, e}$.

Based on these properties, jets have the potential to cause significant particle transport, since they provide a direct path for particles to commute between low and high phase-space density regions. Reporting the formation and discussing the role of jets are the objects of this paper.

The formation process is due mainly to straining between two holes with different velocities. This is illustrated in Fig. 4, which shows snapshots of the electron
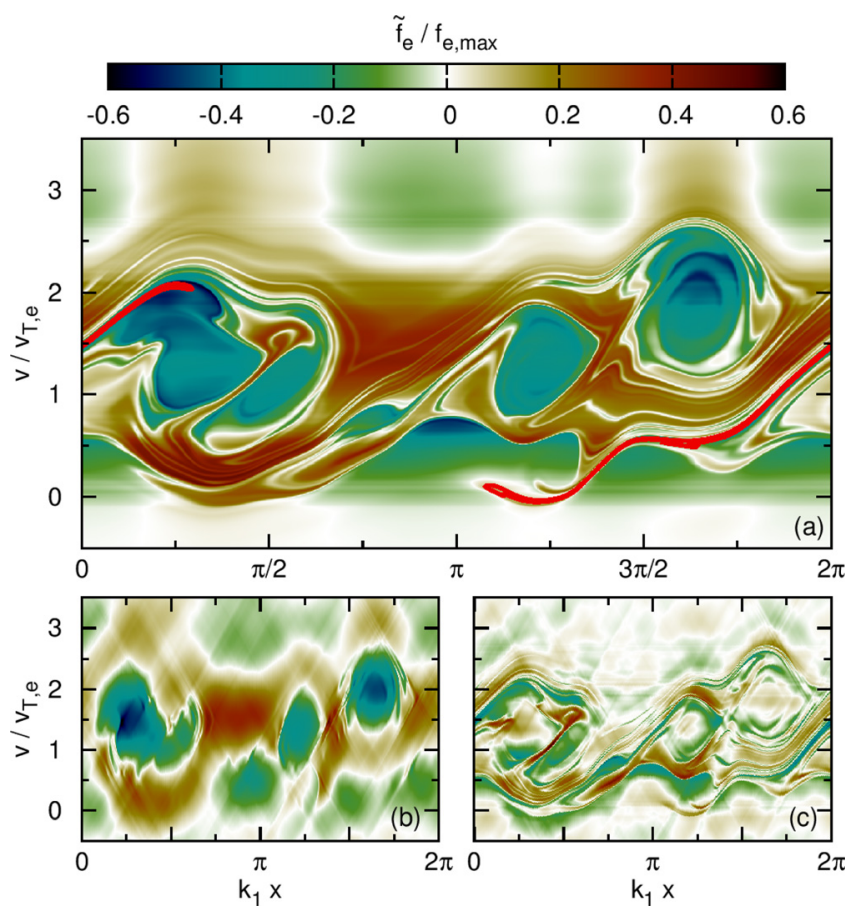

FIG. 3. (a) Snapshot at $\omega_{p, e} t=1180$ of the perturbed electron distribution $\tilde{f}_{e}$, normalized to $f_{e, \max }=f_{e}\left(v_{0, e}\right)$. Solid curve: contour of constant $f_{e} / f_{e, \max }=0.375$. (b) Low-pass filtered $\tilde{f}_{e, \text { holes }}$. (c) High-pass filtered $\tilde{f}_{e, \text { jets }}$. The mass ratio is $m_{i} / m_{e}=4$. The system size is $10 \pi \lambda_{D}$.
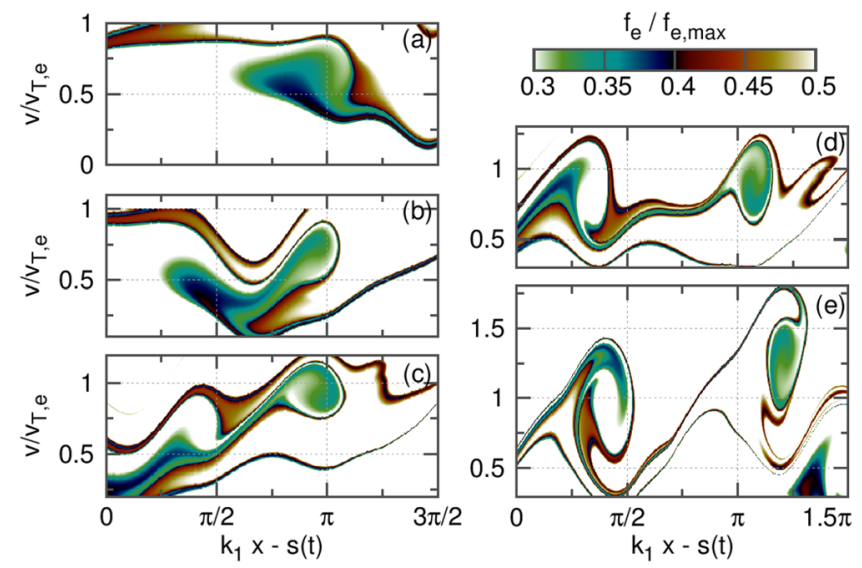

FIG. 4. Jet formation. Snapshots at $\omega_{p, e} t=1100$ (a), 1120 (b), 1140 (c), $1150(\mathrm{~d})$, and 1160 (e) of the electron distribution $f_{e}$ (mass ratio $m_{i} / m_{e}=4$ ). The horizontal coordinate is shifted by $s(t)=0.05 \pi\left(\omega_{p, e} t-1090\right)$ in order to compensate for free-streaming.

distribution function in phase-space, cropped to a small window which follows the birth process. At $t \approx 1100$ [Fig. 4(a)], a blob of negative phase-space density perturbation is extracted from the low-velocity region, attracted by a large hole at higher velocity (not shown in this figure). Since the large hole is traveling faster, the blob does not have time to connect to it. It does however form a hole by itself at about $t \approx 1120$ [Fig. 4(b)], probably because its dimensions approximately satisfy the self-trapping condition. $^{5}$ At $t \approx 1140$ [Fig. 4(c)], a second hole is extracted from the blob, and the bridge between the two holes becomes thinner and thinner. At $t \approx 1150$ [Fig. 4(d)], the two holes are drifting apart. The resulting straining of the bridge is at the heart of the jet formation process. At $t \approx 1150$ [Fig. 4(e)], the bridge has become a jet, satisfying properties P1 (anisotropic) and $\mathrm{P} 2$ (extending from $v=0.3 v_{T, e}$ to $v=1.8 v_{T, e}$ ). It is obvious that any slowly (adiabatically) moving hole forces the ambient passing particles to flow around the hole when the particles come to the separatrix. What is surprising is that this process leads in this case to structures one order of magnitude longer (both in space and in velocity) than the holes.

We turn to analyzing whether such a structure satisfies property P3 (lifetime $\geq$ travel time). Fig. 5 shows a series of snapshots of another typical phase-space jet, namely the one

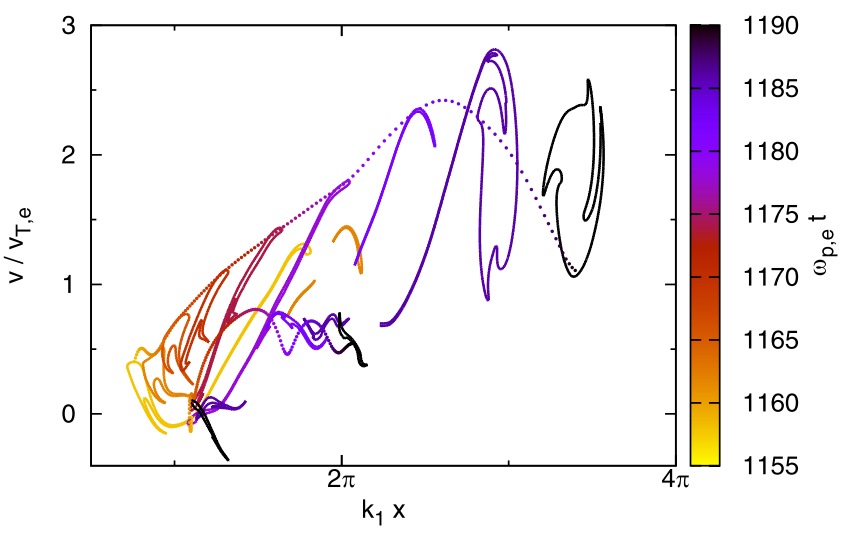

FIG. 5. Time-evolution of a jet. Solid: constant $f_{e} / f_{e \text { max }}=0.375$ contours. Dashed: orbits of two test particles. The mass ratio is $m_{i} / m_{e}=4$. 
shown by a solid curve in Fig. 3(a). Again, properties P1 and $\mathrm{P} 2$ are satisfied. From $t \approx 1160$, the jet grows by straining between a blob of negative phase-space density perturbation in the low-velocity region and a large hole at higher velocity. At $t \approx 1185$, the jet winds around the large hole. At $t \approx 1190$, the jet finally splits into several parts. More precisely, the jet becomes so thin at some locations that it cannot be resolved by the discretized grid in our simulation. In physical experiments, collisions fill the coarse-graining role of a discretized grid. From the jet's formation to its splitting, the jet's lifetime is $\tau_{\text {jet }} \approx 30$. Other jets have shorter $(\approx 10)$ or longer $(\approx 50)$ lifetimes. This is comparable to the average time it takes a particle to change its velocity by $v_{T, e}$, which can be estimated as $\tau_{\text {travel }} \sim v_{T, e} /(e E / m) \sim\left(k_{1} v_{T, e} e \phi / T_{e}\right)^{-1} \sim 25$. Thus, property $\mathrm{P} 3$ is also satisfied. For comparison, a typical hole trapping time is $\tau_{\text {tr }}=2 \pi / \omega_{b} \sim 50$, and a typical hole lifetime is $\tau_{\text {hole }} \sim 500$ or more (and the Kubo number is $K \sim 10$ or more). To summarize, the ordering of time scales is $\tau_{\text {jet }} \sim \tau_{\text {travel }} \sim \tau_{\text {tr }} \ll \tau_{\text {hole }}$.

Particles can ride a jet from low to high velocity or in the opposite direction. Either collisions (here, small numerical collisions are present) or jet splitting make the process irreversible. This yields net velocity-space transport. Fig. 5 includes the orbits of two test particles, which are injected at $t=1158$ on the contour of the jet. We estimate a mean free path in velocity space $\Delta v_{\text {m.f.p. }}$ as the average cumulative change of velocity of 600 such particles during the lifetime of the jet (from $t_{1}=1158$ to $t_{2}=1190$ ), i.e.,

$$
\Delta v_{\text {m.f.p. }}=\left\langle\int_{t_{1}}^{t_{2}}\left|\dot{v}_{i}(t)\right| \mathrm{d} t\right\rangle,
$$

where $i=1 \ldots 600$ is an index of test particles. This procedure yields $\Delta v_{\text {m.f.p. }} / v_{T, e}=2.17$ for this one jet, which indicates that a jet can drive significant stochastic transport. The average non-cumulative change of velocity $\left(\Delta v_{\text {conv. }}\right.$. $\left.=\left\langle v_{i}\left(t_{2}\right)-v_{i}\left(t_{1}\right)\right\rangle\right)$ is $\Delta v_{\text {conv. }} / v_{T, e}=0.48$ for this one jet, which indicates that a jet can also drive convective transport, albeit to a smaller extent. Other jets may convect particles in the opposite direction $\left(\Delta v_{\text {conv. }}<0\right)$. More detailed study is left for future work. To summarize, the ordering of velocity extents is $\left|\Delta v_{\text {conv. }}\right| \lesssim \Delta v_{\text {hole }} \lesssim \Delta v_{\text {m.f.p. }} \sim \Delta v_{\text {jet }} \sim v_{T, e}$.

To quantify jet-driven transport, we split the distribution function into two parts: one containing mainly holes, the other one containing mainly jets. The hole part and jet part are shown in Figs. 3(b) and 3(c), respectively. The jet part is obtained by applying a filter in two-dimensional Fourier space $\left(k_{x}, k_{v}\right)$, where the wave numbers satisfy $k_{v} / k_{x}>R$ and $k_{v}>k_{h}$. Here, $R$ and $k_{h}$ are thresholds, which are empirically adjusted to $\omega_{p, e} R=3$ and $v_{T, e} k_{h}=5$. The remaining part $\left(k_{v} / k_{x} \leq R\right.$ or $\left.k_{v} \leq k_{h}\right)$ is mainly composed of holes. We loosely refer to it as the hole part. We measure velocityspace transport of electron by the flux $\Gamma_{v}(v, t)$, where

$$
\frac{\partial\left\langle f_{e}\right\rangle}{\partial t}+\frac{\partial \Gamma_{v}}{\partial v}=0
$$

Fig. 6 shows the particle flux at $t=1180$. Redistribution, or anomalous resistivity, is driven by a negative (positive) flux in the $v / v_{T, e}>1(<1)$ region. Our main observation is that

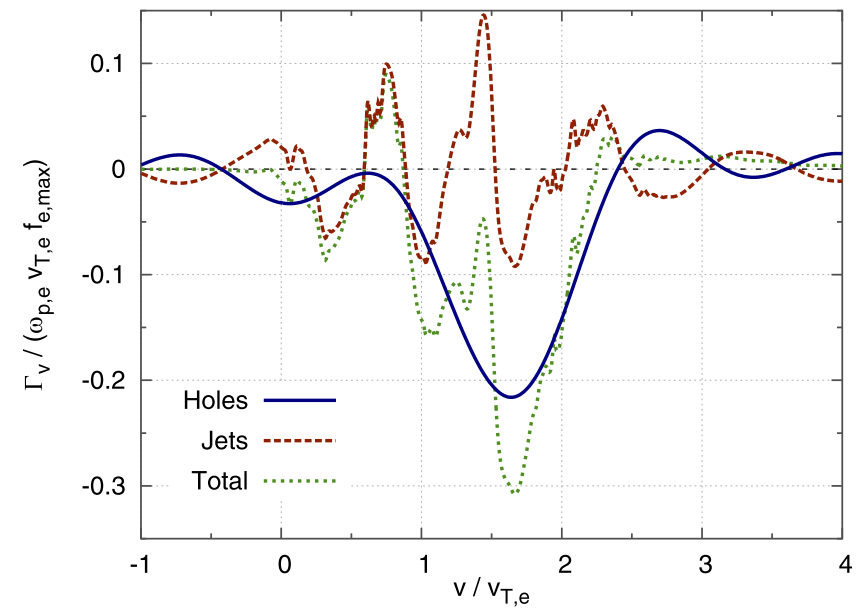

FIG. 6. Velocity-direction particle flux at $\omega_{p, e} t=1180$ (mass ratio $\left.m_{i} / m_{e}=4\right)$.

the contributions of jet part and hole part are of the same order. Jets can either enhance or mitigate redistribution, depending on velocity and time. The oscillations in the holepart and the jet-part for $v / v_{T, e}<0$ and $v / v_{T, e}>2.5$ are an artifact of velocity-space Fourier transformation. We observe that the hole-driven flux is mostly negative, while the jetdriven flux oscillates around zero. This reinforces the intuitive idea that jet transport is essentially stochastic, while hole transport is essentially convective.

Fig. 7 shows the time-evolution of hole-part and jet-part of the particle flux. This figure shows that the above

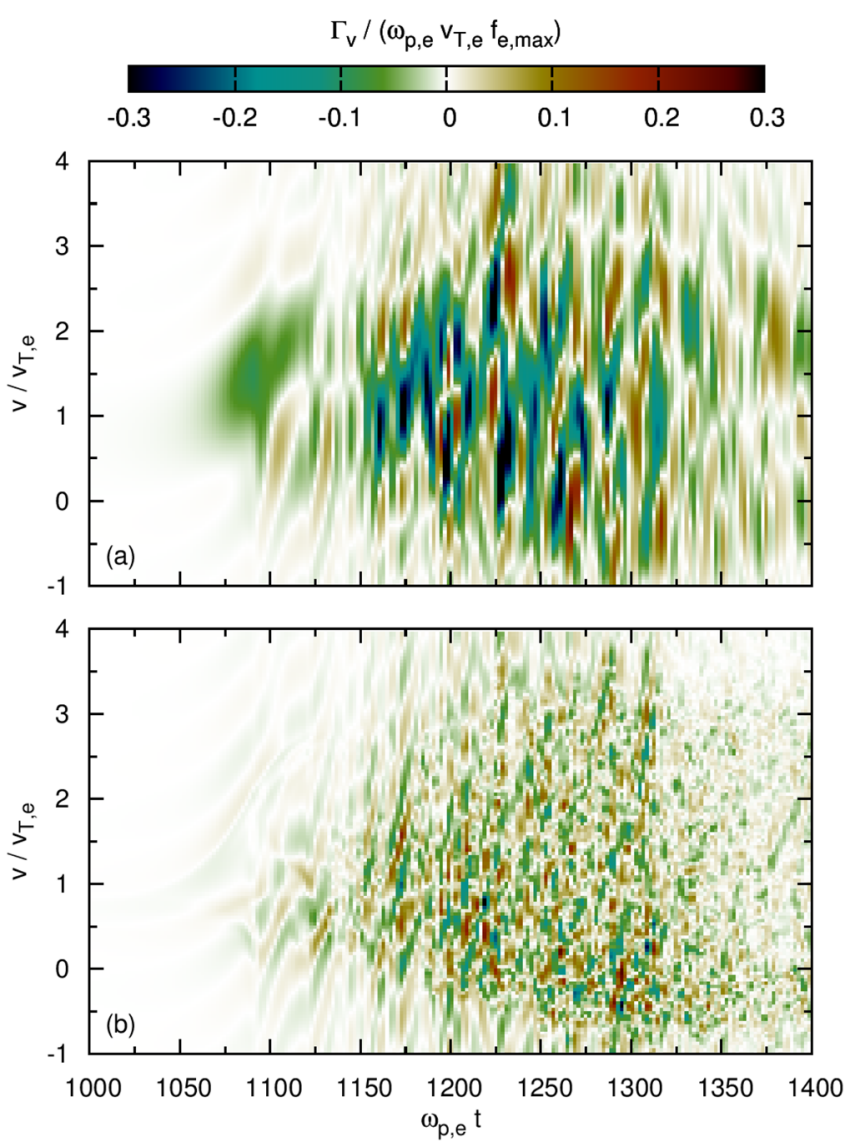

FIG. 7. Time-evolution of the particle flux (mass ratio $m_{i} / m_{e}=4$ ). Contribution from (a) holes and (b) jets. 
observation is not a particular case for $t=1180$. Jet-driven flux is of the same order as hole-driven flux (though slightly smaller) throughout the whole phase of electron redistribution. In the quasi-steady state $(t>1700)$, jets vanish. The electron phase-space is dominated by a single vortex, which results from the merging of the many structures seen in Fig. 3(a).

Globally, jets enhance anomalous resistivity, since they facilitate scattering in velocity-space. However, locally in velocity-time space, jets can mitigate anomalous resistivity. This is the case when holes form a negative gradient in the velocity distribution. As can be seen in Fig. 1, at $t=1180$, the gradient is globally positive, but locally negative (around $v=v_{T, e}$, for example). Thus, flattening the distribution at this location means increasing the electron momentum, which mitigates anomalous resistivity. In an averaged-time viewpoint, though, jets enhance anomalous resistivity.

\section{LARGE MASS RATIO}

In this section, we repeat the above analysis with a mass ratio of $m_{i} / m_{e}=1836$. The system size is $L=2 \pi / k_{1}$, where $k_{1}=0.05 \lambda_{D}^{-1}$. The initial drift is $v_{d}=80 v_{T, i} \approx 1.87 v_{T, e}$. The simulation is performed with $N_{x}=2048$ and $N_{v}=4096$ grid points in configuration-space and velocity-space, respectively, and with a time-step width at most $\Delta t=0.016 \omega_{p, e}^{-1}$.

Fig. 8 shows snapshots of the velocity distribution before, during, and after particle redistribution. As expected, ion redistribution is negligible. Fig. 9 shows the evolution, after the linear growth phase, of mean square potential, and anomalous resistivity.

Fig. 10(a) is a snapshot at $t=2800$ of the perturbed electron distribution. The contour of a jet is overlapped. Because of the large mass ratio, the electrons are easily trapped, and the holes take up most of the electron distribution phasespace. As a consequence, the jets are completely wound around holes, which makes them more difficult to distinguish on a phase-space contour plot. However, our Fourier filter succeeds in extracting them. The hole part and jet part are shown in Figs. 10(b) and 10(c), respectively.

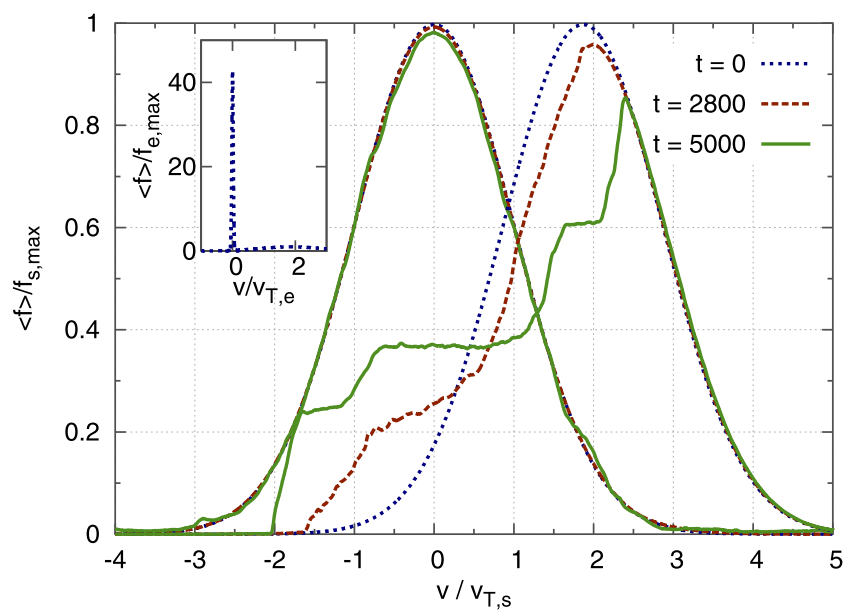

FIG. 8. Snapshots of the ion (left) and electron (right) velocity distributions (mass ratio $m_{i} / m_{e}=1836$ ). Note that ion and electron distributions are not on a same scale. The inset shows them on a same scale.

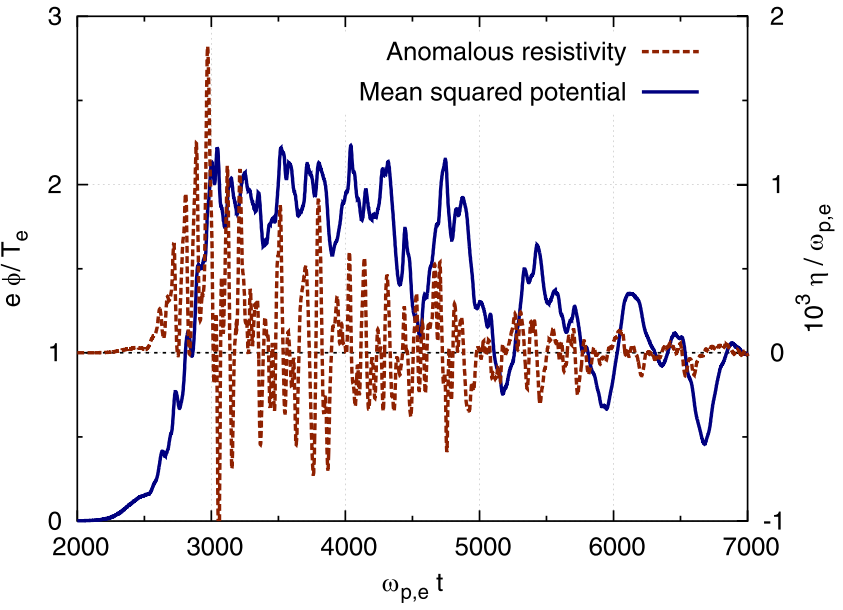

FIG. 9. Time-evolution of the mean square potential (left axis) and anomalous resistivity (right axis). The mass ratio is $m_{i} / m_{e}=1836$. The system size is $40 \pi \lambda_{D}$.

Fig. 11 shows the particle flux at $t=2800$, which roughly corresponds to the peak of electric field amplitude. Again, jet-driven flux is of the same order as holedriven flux. Jets can either enhance or mitigate redistribution, depending on velocity and time. Hole-driven flux is mostly negative, while the jet-driven flux oscillates around zero.

Fig. 12 shows the time-evolution of hole-part and jetpart of the particle flux. This figure shows that the above observation is not a particular case for $t=2800$. Jet-driven flux is of the same order as hole-driven flux (though slightly smaller) throughout the whole phase of electron redistribution.

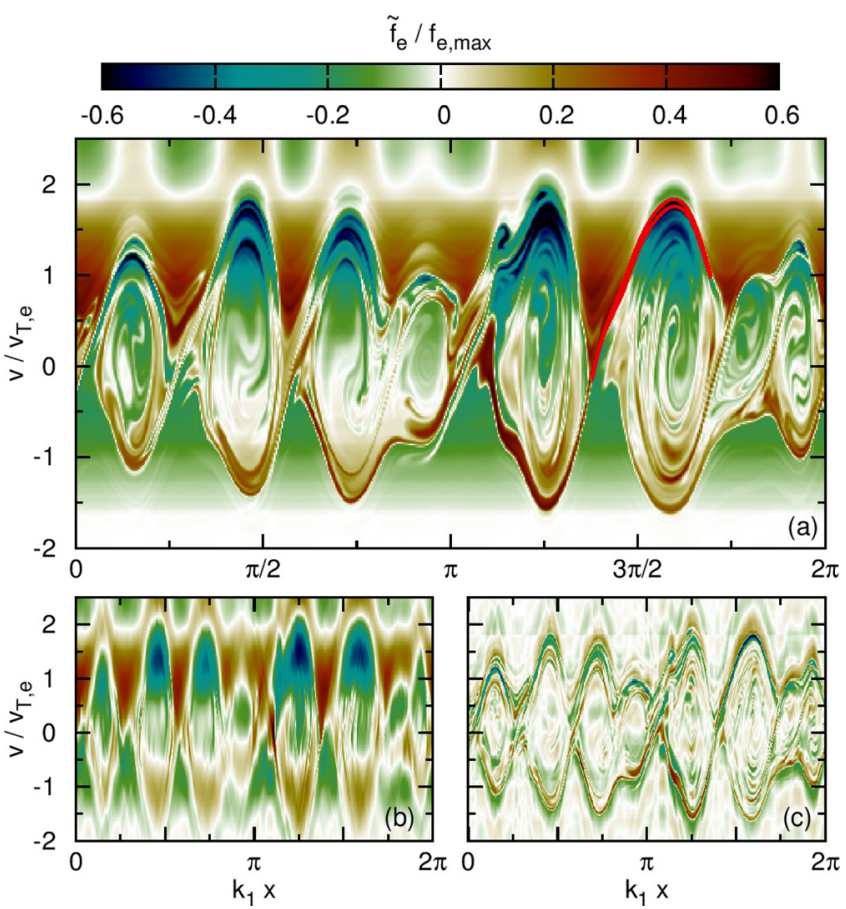

FIG. 10. (a) Snapshot at $\omega_{p, e} t=2800$ of the perturbed electron distribution $\tilde{f}_{e}$, normalized to $f_{e, \max }=f_{e}\left(v_{0, e}\right)$. Solid curve: contour of constant $f_{e} /$ $f_{e, \max }=0.08$. (b) Low-pass filtered $\tilde{f}_{e, \text { holes }}$. (c) High-pass filtered $\tilde{f}_{e, \text { jets }}$. The mass ratio is $m_{i} / m_{e}=1836$. The system size is $40 \pi \lambda_{D}$. 


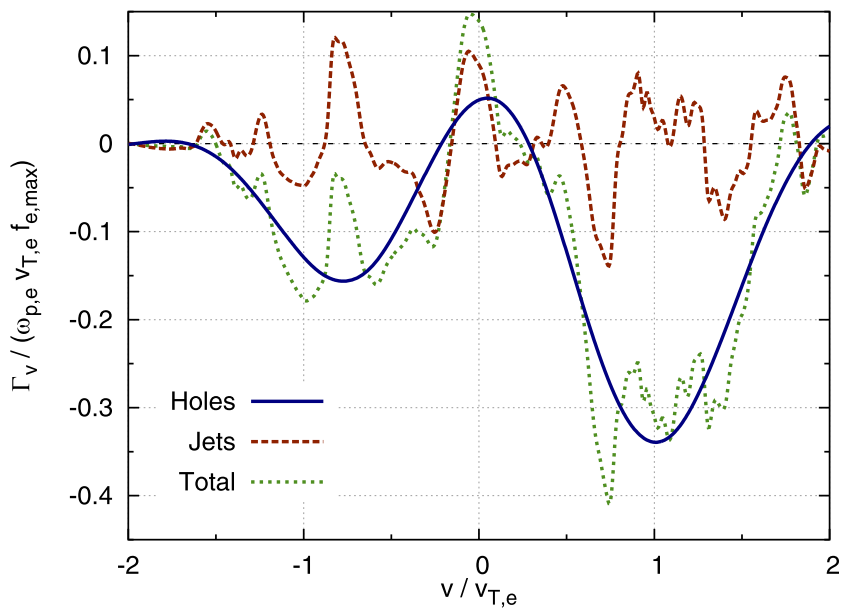

FIG. 11. Velocity-direction particle flux at $\omega_{p, e} t=2800$ (mass ratio $m_{i} /$ $m_{e}=1836$ ).

\section{LARGE SYSTEM SIZE}

A caveat in the above analysis is that the system size is relatively small: comparable to the wavelength of the most unstable wave. For too small system size, quasilinear relaxation is effectively suppressed. Therefore, a question remains: for larger systems, are phase-space jets still relevant or does quasilinear relaxation precede and preclude the formation of such structures? To address this issue, we have performed a

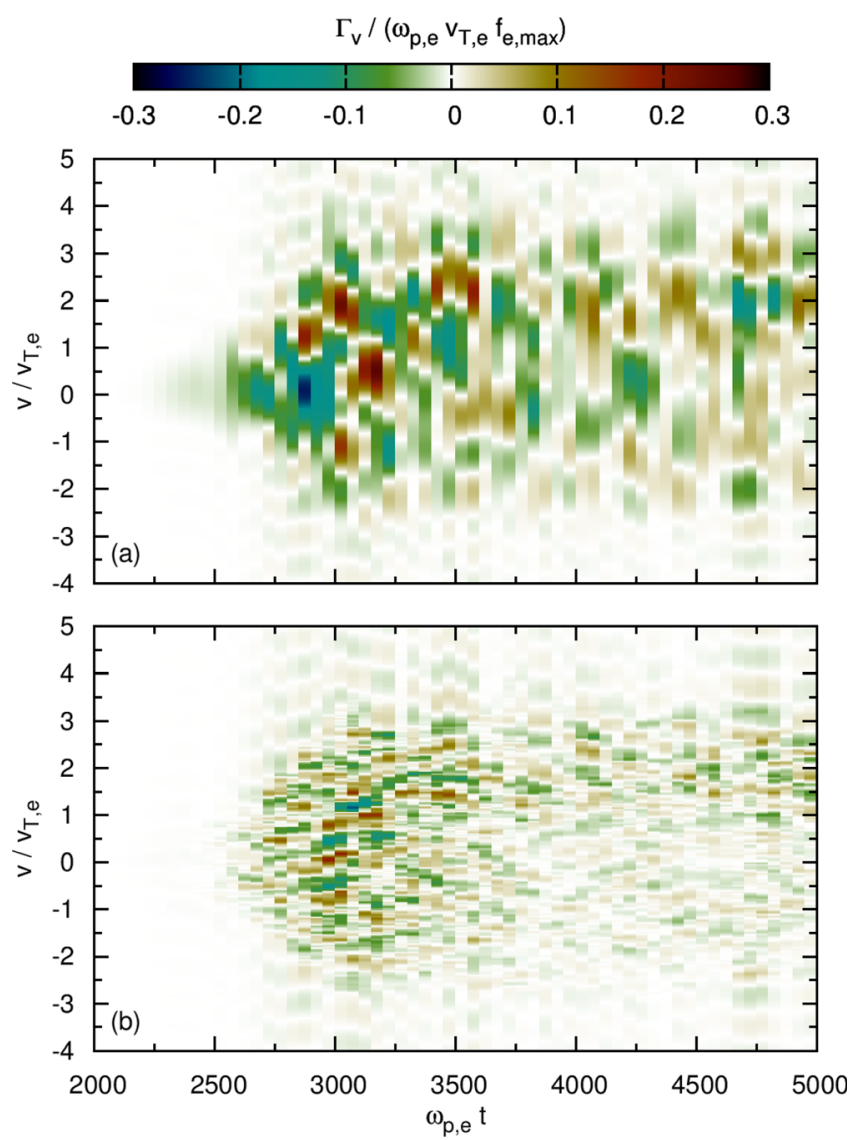

FIG. 12. Time-evolution of particle flux (mass ratio $m_{i} / m_{e}=1836$ ). Contribution from (a) holes and (b) jets. series of simulations with increasing system sizes (and increasing number of grid points $N_{x}$ ).

Fig. 13 shows the time-evolution of electric field amplitude for system size $L=2 \pi / k_{1}$, where $\lambda_{D} k_{1}$ takes three different values, $0.2,0.05$, and 0.01 . We observe that in all three cases, relaxation occurs on a similar timescale. Furthermore, the peak amplitude and the saturated amplitude are similar for 0.05 and 0.01 . However, there is a significant difference between 0.2 and 0.05 . We conclude that the large system size limit corresponds to $\lambda_{D} k_{1}<0.05$.

We have repeated the analysis of Sec. III for $k_{1}=0.01 \lambda_{D}^{-1}$, with $N_{x}=8192$ and $N_{v}=2048$ grid points. We have found qualitatively similar conclusions: phase-space jets are present and account for roughly half of the total particle flux. Fig. 14 is a snapshot at $t=800$ of the perturbed electron distribution. We observe that jets have spatial scales of the order of 10 Debye lengths. Comparison with Figures $3,10,16$, and 18 suggests that this is true regardless of the mass ratio, system size, or whether the system is driven or decaying.

\section{DRIVEN SYSTEM}

An other caveat in the above analysis is that we have studied an initial value problem. An other question remains: are phase-space jets (and holes) still relevant when an external drive supplies free energy continuously? To address this issue, we have studied an other set of simulations, where the initial velocity drift between ion and electron is zero $\left(v_{d}=0\right)$, and the system is driven by a constant and uniform external electric field $E_{\text {ext }}$.

Fig. 15 shows the time-evolution of mean square potential and anomalous resistivity in a system driven by an external electric field $E_{\text {ext }}=10^{-4} E_{\text {norm }}$, where $E_{\text {norm }}=m_{e} v_{T, e} \omega_{p, e} / e$. We observe that the peak amplitude, peak anomalous resistivity, and timescale of relaxation are comparable to the case with initial drift and no drive. Further in time, as the constant electric field continues to provide free energy in the form of a current, more relaxation events occur, such as for $\omega_{p, e} t \approx 10500$.

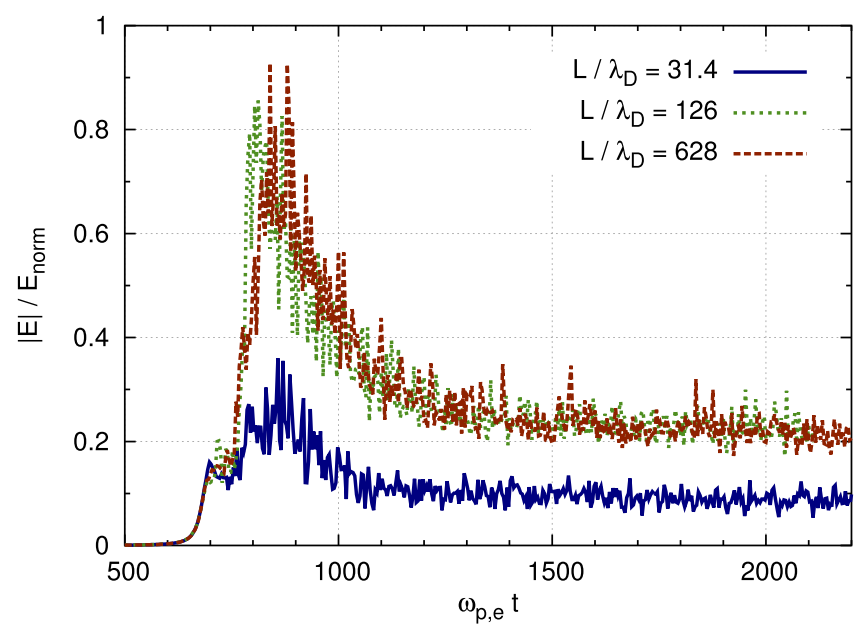

FIG. 13. Time-evolution of electric field amplitude, normalized by $E_{\text {norm }}=m_{e} v_{T, e} \omega_{p, e} / e$ for different system sizes, which are indicated in the legend. Mass ratio is $m_{i} / m_{e}=4$. Initial drift is $v_{d}=2.1 v_{T, e}$. 


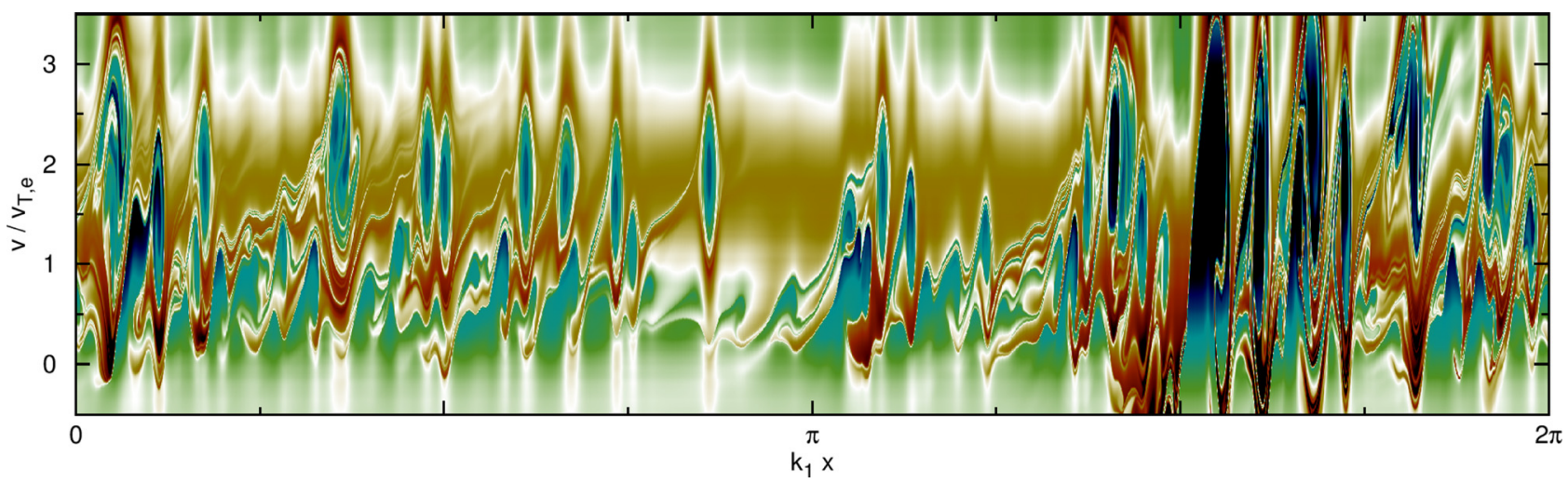

FIG. 14. Snapshot at $\omega_{p, e} t=800$ of the perturbed electron distribution $\tilde{f}_{e}$, normalized to $f_{e, \max }=f_{e}\left(v_{0, e}\right)$. The system size is $200 \pi \lambda_{D}$.

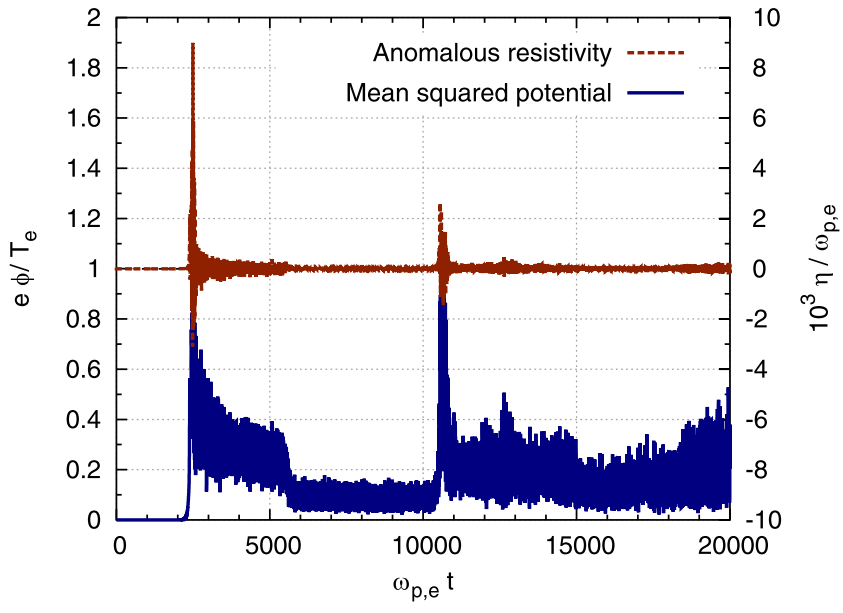

FIG. 15. Time-evolution of the mean square potential (left axis) and anomalous resistivity (right axis) in a system driven by an external electric field. The mass ratio is $m_{i} / m_{e}=4$.

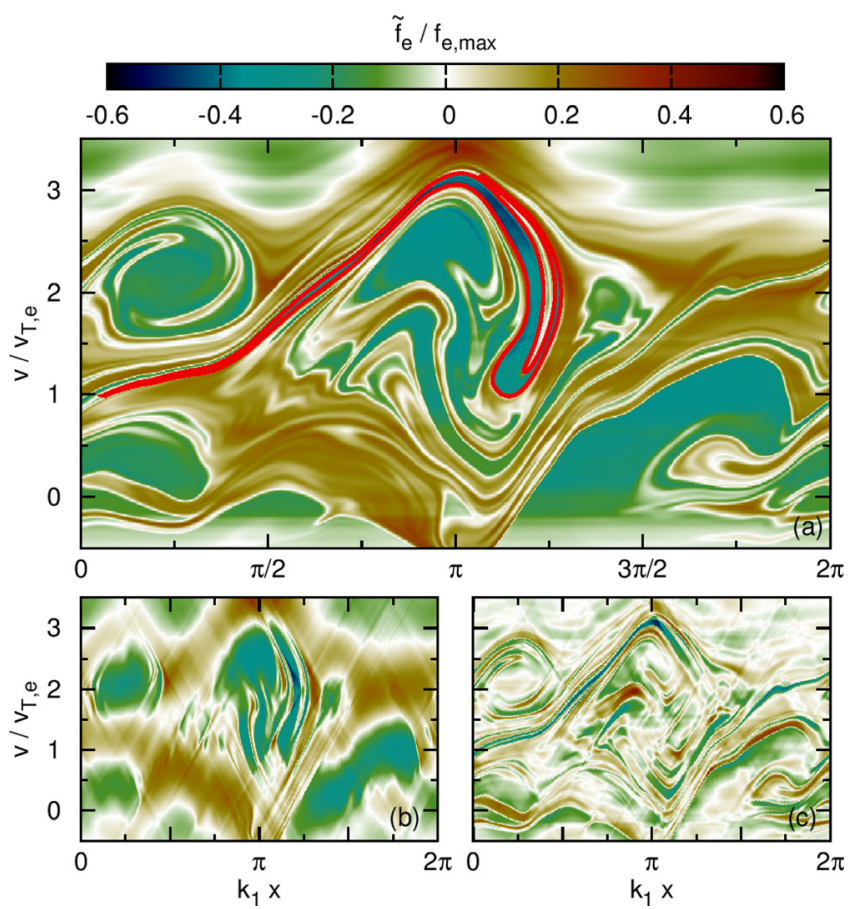

FIG. 16. (a) Snapshot at $\omega_{p, e} t=2480$ of the perturbed electron distribution $\tilde{f}_{e}$, normalized to $f_{e, \max }=f_{e}\left(v_{0, e}\right)$, for a driven system. Solid curve: contour of constant $f_{e} / f_{e, \max }=0.5$. (b) Low-pass filtered $f_{e \text { holes }}$. (c) High-pass filtered $\tilde{f}_{e \text {.jets. }}$ The mass ratio is $m_{i} / m_{e}=4$.

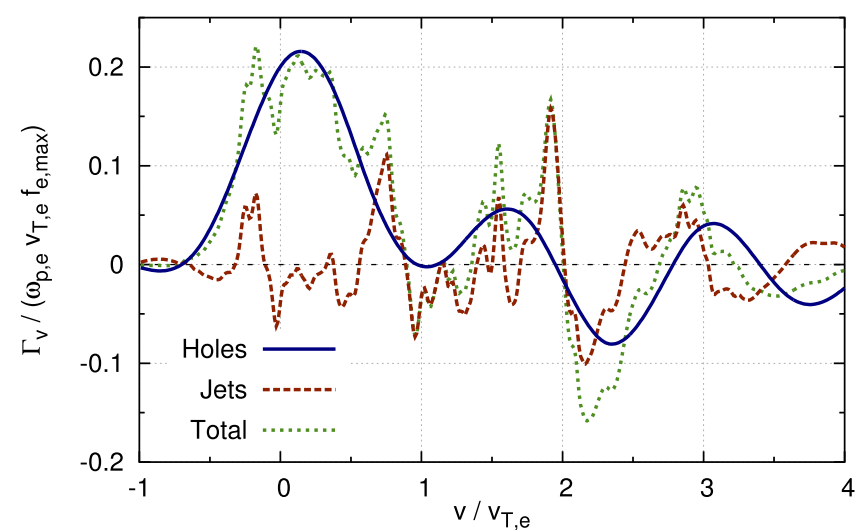

FIG. 17. Velocity-direction particle flux at $\omega_{p, e} t=2480$ (mass ratio $m_{i} /$ $m_{e}=4$, driven system).
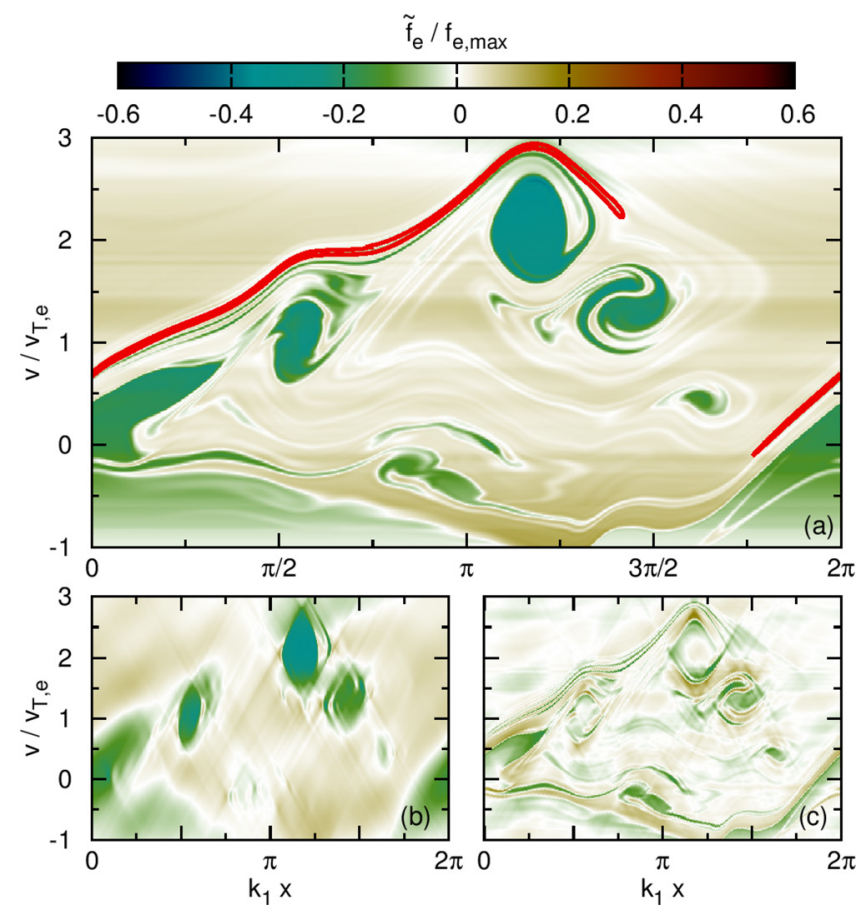

FIG. 18. Snapshot at $\omega_{p, e} t=10560$ of the perturbed electron distribution $\tilde{f}_{e}$, normalized to $f_{e \max }=f_{e}\left(v_{0, e}\right)$, for a driven system. The mass ratio is $m_{i} /$ $m_{e}=4$. 


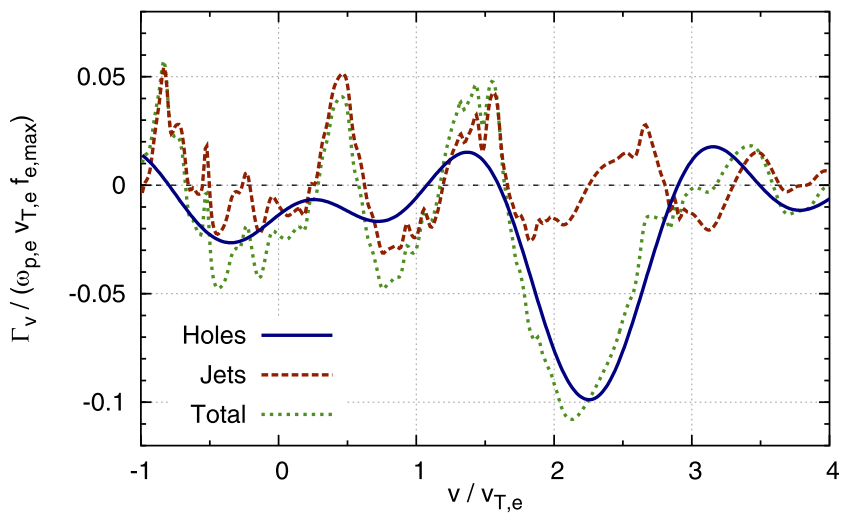

FIG. 19. Velocity-direction particle flux at $\omega_{p, e} t=10560$ (mass ratio $m_{i} /$ $m_{e}=4$, driven system).

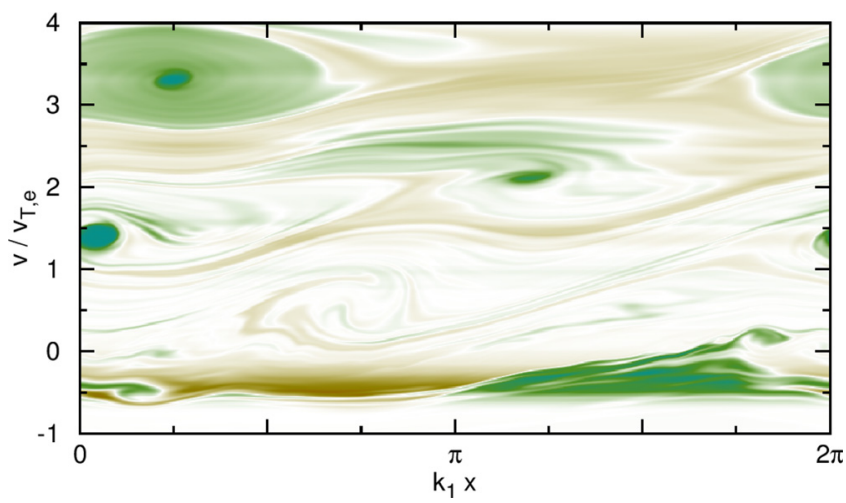

FIG. 20. Snapshot at $\omega_{p, e} t=20000$ of the perturbed electron distribution $\tilde{f}_{e}$, normalized to $f_{e, \max }=f_{e}\left(v_{0, e}\right)$, for a driven system.

We repeat our analysis of phase-space structures and their impact on particle flux for the first and the second relaxation events, which occur at $\omega_{p, e} \approx 2480$ and 10560 , respectively.

Let us repeat our analysis of phase-space structures and their impact on particle flux for this driven case. Fig. 16(a) is a snapshot of the perturbed electron distribution at $\omega_{p, e} t=2480$, which roughly corresponds to the peak of electric field amplitude. The contour of a jet is overlapped. The hole part and jet part are shown in Figs. 16(b) and 16(c), respectively. Fig. 17 shows the particle flux at $\omega_{p, e} t=2480$. Similar figures, Figs. 18 and 19, are provided for the second redistribution event at $\omega_{p, e} t=10560$. Again, for both relaxation events, jet-driven flux is of the same order as holedriven flux.

We measure the dominant mode frequency as $\omega_{I A}=0.123 \omega_{p, e}$. Therefore, the second peak corresponds to $\omega_{I A} t=1295$. On longer timescales, the jets are not expected to play any significant role, except during transient redistribution bursts. Fig. 20 is a snapshot of the perturbed electron distribution at $\omega_{p, e} t=20000\left(\omega_{I A} t=2450\right)$, which corresponds to a quiescent period between bursts. We observe that the jets are absent except for faint remnants. The phasespace dynamics is dominated by holes, which are much more robust. However, the anomalous resistivity during these quiescent periods is negligible (compared to during bursts), so this does not undermines out conclusion that jets contribute to about half of anomalous resistivity.

\section{CONCLUSION}

In summary, velocity-space redistribution and anomalous resistivity are due to phase-space turbulence, which includes not only holes, but also phase-space jets. The latter are highly anisotropic structures, with an extent in velocity of the order of the electron thermal velocity. Compared to phase-space holes, which are very robust structures, jets are relatively transient objects. However, we showed that jets survive long enough for particles to scatter between low and high phase-space density regions, and drive significant particle redistribution. Jets and holes are associated with fundamentally different transport processes. Hole-driven transport is essentially convective, since trapped particles accelerate along with the hole. Jet-driven transport is essentially stochastic, since particles may accelerate or decelerate along the jet. These conclusions stand for both initial value and driven systems. When the mass ratio is high, ion relaxation is negligible, and jets are completely wound around holes. Since jets emerge from holes, jets are expected to play an important role in the $K \gg 1$ (strong wave-particle interactions) regime, or in other words, when the collision meanfree-path is much larger than the wavelengths of unstable modes.

Although we treated in this paper the example of the ion-acoustic wave in 1D plasma with $T_{i}=T_{e}$, we have carried additional simulations with other parameters, including a subcritical case (velocity drift below linear threshold), ${ }^{38}$ and other models and other waves. Based on these, we expect qualitatively similar conclusions for many regimes of strong wave-particle interactions in dissipative collisionless or quasi-collisionless plasmas.

\section{ACKNOWLEDGMENTS}

The authors are grateful for stimulating discussions with K. Itoh, S.-I. Itoh, C. Nguyen, Y. Idomura, X. Garbet, and the participants in the 2009, 2011, and 2013 Festival de Théorie. This work was supported by three grants-in-aid for scientific research of JSPS, Japan (21224014, 23244113, and 25887041), by the collaboration program of the RIAM of Kyushu University and Asada Science Foundation, by the WCI Program of the NRF of Korea funded by the Ministry of Education, Science and Technology of Korea [WCI 2009001], and by CMTFO via U.S. DoE Grant No. DE-FG0204ER54738. Computations were performed on the Plasma Simulator at NIFS.

${ }^{1}$ H. Schamel, Phys. Rep. 140, 161 (1986).

${ }^{2}$ J. C. Mcwilliams, J. Fluid Mech. 146, 21 (1984).

${ }^{3}$ M. Lesur and P. H. Diamond, Phys. Rev. E 87, 031101 (2013).

${ }^{4}$ H. Schamel, Phys. Plasmas 19, 020501 (2012).

${ }^{5}$ T. H. Dupree, Phys. Fluids 25, 277 (1982).

${ }^{6}$ C. T. Hsu, C. Z. Cheng, P. Helander, D. J. Sigmar, and R. White, Phys. Rev. Lett. 72, 2503 (1994).

${ }^{7}$ T. H. Dupree, Phys. Rev. Lett. 25, 789 (1970).

${ }^{8}$ B. Eliasson and P. Shukla, Phys. Rep. 422, 225 (2006).

${ }^{9}$ J. F. Drake, M. Swisdak, C. Cattell, M. A. Shay, B. N. Rogers, and A Zeiler, Science 299, 873 (2003).

${ }^{10}$ P. H. Sakanaka, Phys. Fluids 15, 304 (1972).

${ }^{11}$ H. Mynick and N. Pomohrey, Nucl. Fusion 34, 1277 (1994).

${ }^{12}$ H. Biglari, P. H. Diamond, and P. W. Terry, Phys. Fluids 31, 2644 (1988). 
${ }^{13}$ P. H. Diamond, S.-I. Itoh, and K. Itoh, Modern Plasma Physics: Volume 1, Physical Kinetics of Turbulent Plasmas, Modern Plasma Physics (Cambridge University Press, Cambridge, 2010).

${ }^{14}$ Y. Kosuga and P. H. Diamond, Phys. Plasmas 18, 122305 (2011).

${ }^{15}$ Y. Kosuga, S.-I. Itoh, P. H. Diamond, K. Itoh, and M. Lesur, Plasma Fusion Res. 9, 3403018 (2014). Also available at http://www.jspf.or.jp/ PFR/PFR_articles/pfr2014S2/pfr2014_09-3403018.html

${ }^{16}$ K. V. Roberts and H. L. Berk, Phys. Rev. Lett. 19, 297 (1967).

${ }^{17}$ K. Saeki, P. Michelsen, H. L. Pécseli, and J. J. Rasmussen, Phys. Rev. Lett. 42, 501 (1979).

${ }^{18}$ H. Schamel, Phys. Scr. 20, 336 (1979).

${ }^{19}$ R. H. Berman, D. J. Tetreault, and T. H. Dupree, Phys. Fluids 28, 155 (1985).

${ }^{20}$ H. L. Berk, B. N. Breizman, and N. V. Petviashvili, Phys. Lett. A 234, 213 (1997).

${ }^{21}$ H. L. Berk, B. N. Breizman, J. Candy, M. Pekker, and N. V. Petviashvili, Phys. Plasmas 6, 3102 (1999).

${ }^{22}$ M. K. Lilley, B. N. Breizman, and S. E. Sharapov, Phys. Plasmas 17, 092305 (2010).

${ }^{23}$ R. Nyqvist, M. Lilley, and B. Breizman, Nucl. Fusion 52, 094020 (2012).

${ }^{24}$ M. Lesur, Phys. Plasmas 20, 055905 (2013).
${ }^{25}$ T. H. Dupree, Phys. Fluids 15, 334 (1972).

${ }^{26}$ T. Boutros-Ghali and T. H. Dupree, Phys. Fluids 25, 874 (1982).

${ }^{27}$ H. L. Berk, C. E. Nielsen, and K. V. Roberts, Phys. Fluids 13, 980 (1970).

${ }^{28}$ M. M. Oppenheim, G. Vetoulis, D. L. Newman, and M. V. Goldman, Geophys. Rev. Lett. 28, 1891 (2001).

${ }^{29}$ R. H. Berman, D. J. Tetreault, T. H. Dupree, and T. Boutros-Ghali, Phys. Rev. Lett. 48, 1249 (1982)

${ }^{30}$ M. Lesur, Y. Idomura, and X. Garbet, Phys. Plasmas 16, 092305 (2009).

${ }^{31}$ Y. Omura, H. Matsumoto, T. Miyake, and H. Kojima, J. Geophys. Res. 101, 2685, doi:10.1029/95JA03145 (1996).

${ }^{32}$ C. E. J. Watt, R. B. Horne, and M. P. Freeman, Geophys. Res. Lett. 29, 4, doi:10.1029/2001GL013451 (2002).

${ }^{33}$ P. Petkaki, C. E. J. Watt, R. B. Horne, and M. P. Freeman, J. Geophys. Res. 108, A12, doi:10.1029/2003JA010092 (2003).

${ }^{34} \mathrm{~W}$. Oohara and R. Hatakeyama, Phys. Rev. Lett. 91, 205005 (2003).

${ }^{35}$ R. G. Greaves and C. M. Surko, Phys. Rev. Lett. 75, 3846 (1995).

${ }^{36} \mathrm{H}$. Schamel and J. Korn, Phys. Scr. 1996(T63), 63 (1996).

${ }^{37}$ T. H. Dupree, Phys. Fluids 26, 2460 (1983).

${ }^{38}$ M. Lesur, P. H. Diamond, and Y. Kosuga, Plasma Phys. Controlled Fusion 56, 075005 (2014). 